\title{
Urgencias en dermatología
}

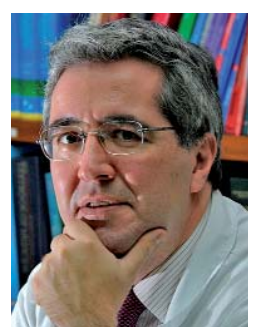

Ricardo Suárez Fernández Jefe del Servicio de Dermatología. Hospital General Universitario Gregorio Marañón. Madrid

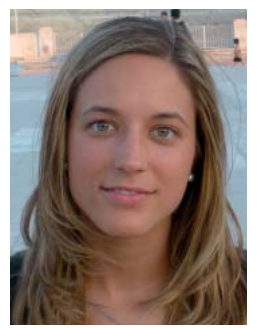

Paloma Borregón Nofuentes

Médico residente de Dermatología, 4.ำ año. Hospital General Universitario Gregorio Marañón. Madrid
El concepto de emergencia en dermatología implica aquella situación patológica con riesgo vital inmediato para el paciente. Evidentemente, no hay muchas, pero las hay: sepsis de origen cutáneo, infecciones graves, necrólisis epidérmica tóxica (fig. 1), psoriasis pustulosa eritrodérmica (fig. 2), enfermedades ampollosas generalizadas y algún cuadro más que suponga una insuficiencia cutánea aguda (fig. 3). Otro concepto es el de urgencia dermatológica, que es la que implica que el paciente demande atención inmediata. Y aquí sabemos todos que hay muchísimas, aunque no constituyan una situación de emergencia. Un 6-10\% de los pacientes que acuden a un Servicio de Urgencias lo hacen por dermopatías, con trastornos de lo más variados: infecciones, eccemas, urticarias, reacciones cutáneas por fármacos, picaduras y un amplio abanico de procesos, como dermatosis neutrofílicas, psoriasis, dermatitis de contacto o atópica, e incluso afecciones tumorales, enfermedades de transmisión sexual y su equivalente de venereofobia, etc. Hay referencias bibliográficas que afirman, como era de esperar, que las afecciones cutáneas no se tratan adecuadamente si no es por dermatólogos y reconocen una escasa correlación entre el diagnóstico de presunción de la dermopatía por los médicos no especialistas y el diagnóstico final ${ }^{1}$. Esta afirmación conlleva, entre otras conclusiones, una incorrecta derivación a Urgencias de

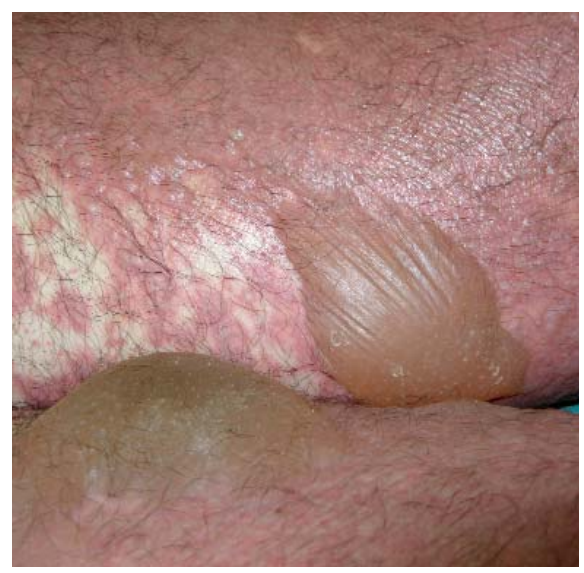

Figura 1. Necrólisis epidérmica tóxica.

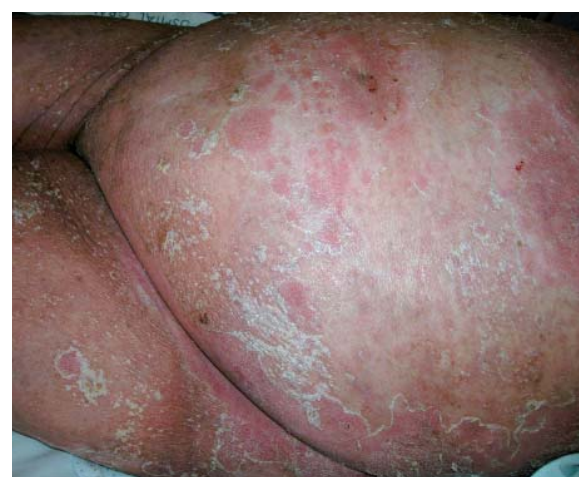

Figura 2. Psoriasis pustuloso.

hasta un $24 \%^{2}$. Datos publicados en España encuentran un 10\% de casos dermatológicos en Urgencias de Pediatría en el Hospital Pediátrico Valle de Hebrón, con una tasa de ingreso del $6 \%$, cifra considerable que apoya la necesidad del dermatólogo en un Servicio de Urgencias.

Desde hace unos años, se organiza el curso de «Urgencias en dermatología» en el Hospital Gregorio Mara- 


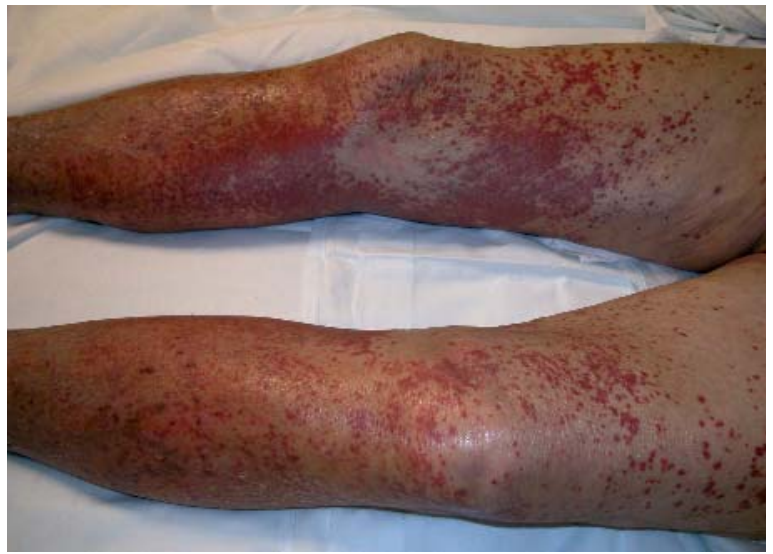

Figura 3. Vasculitis de tipo Schonlein Henoch.

ñón de Madrid, con una asistencia cada año mayor. En principio, se creó para dermatólogos jóvenes, médicos de urgencias o de asistencia primaria interesados, pero la verdad es que la importante asistencia nos demostró la gran demanda de este curso. El médico que atiende las frecuentes urgencias en dermatología se enfrenta a diferentes enfermedades, como infecciones víricas, bacterianas, parasitarias, procesos inflamatorios de todo tipo, exantemáticos o subagudos, cuyo mal diagnóstico previo ha supuesto la comprensible preocupación del paciente ante una posible enfermedad grave. En niños y embarazadas, esta preocupación se agudiza. En el Congreso Nacional de Dermatología de 2011 en Santiago de Compostela (España), el taller práctico de urgencias para residentes tuvo un lleno total, con imposibilidad de atender a todos los asistentes. Las situaciones urgentes dermatológicas suponen, por lo tanto, un importante motivo de demanda formativa entre los médicos que se enfrentan de forma habitual con estas enfermedades.

La duda siempre aparece cuando se plantea si las urgencias dermatológicas deben ser atendidas en Servicios de Urgencia hospitalarios, por médicos internos residentes (MIR) o incluso por médicos adjuntos. Personalmente, creo que, en un estado de bienestar social como el que, de momento hasta ahora, predomina en nuestro entorno, sí se debe prestar este servicio a la sociedad, pues- to que puede suponer una ayuda vital en el manejo de algún paciente (exantemas con especificidad, como fiebre botonosa, meningococemia, sepsis gonocócica, herpes diseminado, mucor etc.), y la posibilidad de salvar una sola vida ya justifica su existencia. Por otra parte, y de forma más pragmática, estos pacientes acuden a una demanda de atención dermatológica que, si no se maneja con habilidad, puede suponer demoras en la sanidad, mayor tiempo de estancia en Urgencias y, en general, aumento del gasto sanitario por falta de resolución ante el problema presentado. Un tercer punto es el formativo, ya que el Consejo Nacional de la Especialidad incluye las guardias de dermatología para MIR desde su segundo año de residencia y, si este punto no lo cumplimos, quizás estaríamos formando peores especialistas. Es evidente que la enfermedad dermatológica urgente (exantemas, urticaria, dermatosis neutrofílicas, viriasis, etc.) no se ve con tanta frecuencia en servicios sin atención urgente. Por supuesto, si, como es cada vez más generalizado, el Servicio de Dermatología realiza intervenciones de cirugía mayor ambulatoria, debe estar presente ante posibles complicaciones hemorrágicas, dehiscentes, etc. en estos pacientes.

En el Hospital Gregorio Marañón, también hemos recogido la casuística de los pacientes atendidos por nosotros en el Servicio de Urgencias durante 3 meses, para definir las características epidemiológicas del paciente que demanda asistencia dermatológica de urgencia y evaluar la naturaleza de los procesos que lo motivan a consultar, así como para dar consistencia a la idea de la necesidad de un especialista que atienda la enfermedad dermatológica de urgencia. Recogimos datos de los pacientes mayores de 16 años que fueron asistidos por un residente de Dermatología de segundo a cuarto año en horario de 10 a 22 horas.

El paciente asistido por el Servicio de Dermatología en Urgencias del Hospital Gregorio Marañón es con mayor frecuencia mujer, se encuentra en el intervalo de edad entre 26 y 40 años y no tiene antecedentes personales de interés. Este 
resultado es similar al descrito por Gonzalez et al. en $2001^{3}$.

Los pacientes presentaron gran dispersión de edad. La media fue de 46 años, más alta que en algunos estudios anteriores ${ }^{4,5}$, pero debe tenerse en cuenta que los menores de 16 años no fueron incluidos en nuestro estudio y la diferencia puede deberse a ello. En cuanto al sexo, todos los estudios $^{1-11}$ concluyen como el nuestro, que consultan más mujeres, con porcentajes similares al encontrado por nosotros (53\% mujeres y $47 \%$ hombres). Un dato que llama la atención y que ya se ha descrito es que las mujeres consultan cuando las lesiones tienen menos tiempo de evolución, probablemente debido a motivos estéticos que llevan a una percepción más alarmante de su afección dermatológica, pero nosotros no encontramos diferencias estadísticamente significativas.

Con respecto a la procedencia, menos de la mitad de los pacientes que atendimos pertenecían al Gregorio Marañón (46\%), mientras que el resto $(54 \%)$ eran de otros hospitales que, en su mayoría, no tienen dermatólogo en Urgencias.

La mitad de los pacientes consultaron cuando el cuadro cutáneo tenía menos de 1 semana de evolución, pero hasta un $13 \%$ acude a Urgencias teniendo las lesiones desde hace más de 6 semanas. Muchos de estos pacientes $(11 \%)$ ya tenían cita pendiente con Dermatología pero, debido a las largas listas de espera de nuestra especialidad, acudieron a Urgencias por algo que ya no era realmente urgente, haciendo un mal uso de los recursos sanitarios, como ya describió Ribera ${ }^{6}$.

En nuestro estudio, la localización más frecuente de aparición de lesiones fueron las extremidades, seguidas de tronco y cabeza. En cuanto a los diagnósticos, para facilitar la recogida de datos, agrupamos los diagnósticos en 10 categorías, que son las que con mayor frecuencia se ven en la urgencia (tabla 1). De ellas, las infecciones y los eccemas suponen más de la mitad (infecciones $32 \%$, eccemas $20 \%$ ) y además constituyen las enfermedades más frecuentes en todos los grupos de edad. Estos datos han sido recogidos por numerosos autores.
Tabla 1. Distribución de enfermedades en 10 grandes grupos.

\begin{tabular}{l|c}
\hline Diagnóstico & Total (\%) \\
\hline Infecciones & 32 \\
\hline Eccema & 20 \\
\hline Otros & 10 \\
\hline Pendiente de estudio & 9,3 \\
\hline $\begin{array}{l}\text { Reagudización de enfermedades } \\
\text { crónicas }\end{array}$ & 8,2 \\
\hline Agentes físicos & 5,3 \\
\hline Urticaria & 5,2 \\
\hline Fármacos & 3,5 \\
\hline Vasculitis & 3,5 \\
\hline Tumores & 3 \\
\hline Total & 100 \\
\hline
\end{tabular}

Gonzalez et al. ${ }^{3}$ describen la urticaria como el proceso individual más frecuente, pero nosotros encontramos como más frecuente el eccema agudo (20\%) y después el herpes zóster, la celulitis y la erisipela; la urticaria se observa solamente en un 5,2\% de los casos. Emitimos 57 diagnósticos diferentes, cosa que demuestra que existe un porcentaje de pacientes que padece enfermedades de baja prevalencia, lo que supone una dificultad diagnóstica muy elevada para el médico no dermatólogo. Herrera et al. ${ }^{7}$ también apoyan esta afirmación.

El 76,3\% de los pacientes fueron diagnosticados y tratados sin necesidad de realizar ninguna prueba complementaria, dato que demuestra que tener un dermatólogo en Urgencias no solo mejora la calidad de la asistencia, sino que además es rentable, dado que apenas consume recursos y, con la clínica, puede orientar el diagnóstico.

En atención primaria, Valcuende et al. ${ }^{8}$ vieron que solo el 13,4\% de los pacientes dermatológicos atendidos eran derivados a un dermatólogo. Sin embargo, nosotros remitimos para seguimiento, o bien a nuestra consulta en el hospital, o bien al dermatólogo de zona para seguimiento o estudio, al 43\%, lo que traduce una mayor calidad asistencial para el enfermo dermatológico. Nosotros ingre- 
samos al $5 \%$ de los pacientes, cifra superior a la de estudios previos.

Así pues, con los datos aportados y junto a otros estudios $^{9-12}$, podemos afirmar que las urgencias dermatológicas son rentables para el sistema sanitario, eficientes, formativas y muy recomendables para una asistencia sanitaria adecuada ${ }^{13}$.

\section{BIBLIOGRAFÍA}

1. Valcuende F, Ferraz C, Tomás G, Pitarch A, Almela T, Bertoméu F. Análisis de las urgencias dermatológicas en el servicio de urgencias de un hospital comarcal. Actas Dermosifiliogr. 1996;87:305-9.

2. López JL, Argila D. Urgencias en dermatología. Arch Dermatol. 1996;7:54.64.

3. González Ruiz A, Bernal Ruiz Al, García Muñoz M, Miranda Romero A, Castrodeza Sanz J. Urgencias dermatológicas en un hospital de referencia. Actas Dermosifilogr. 2001;92:342348.

4. García JM, Cageao C, Del Pozo J, Almagro M, Martínez W, Fonseca E. Estudio de las consultas dermatológicas ambulatorias urgentes en el Área Sanitaria de La Coruña. Piel. 1997; 12:233-240.
5. Suárez R. Urgencias en dermatología. Piel. 2009;24:789-90.

6. Ribera M. Urgencias en dermatología. Actas Dermosifiliogr. 1997;88:353-7.

7. Herrera M, Calvente MJ, Del Cerro M, Rueda M, Fernández C, Robledo A. Urgencias en dermatología. Estudio descriptivo. Actas Dermosifiliogr. 1996;76:675-80.

8. Valcuende F, Almela T, Iglesias JD, Pitarch A, Tomás G, Bertoméu F. Urgencias dermatológicas en atención primaria. Actas Dermosifiliogr. 1997;88:327-32.

9. Elcuaz R, Beorlegui J, Cortés F, Goñi C, Espelosín G, Sagredo T. Análisis de las derivaciones urgentes a dermatología. Aten Primaria. 1998;21:131-6.

10. González A, Miranda A, Sánchez P, Martínez M, Cuadrado C, Samaniego $E$, et al. Urgencias en dermatología. Actas Dermosifiliogr. 1998;89:350-3.

11. Gupta S, Sandhu K, Kumar B. Evaluation of emergency dermatological consultations in a tertiary care centre in North India. J Eur Acad Dermatol Venereol. 2003;17:303-5.

12. Gil MP, Velasco M, Miguel J, Quecedo E, Martínez JA, Nagore $\mathrm{E}$, et al. Análisis de las urgencias dermatológicas de un hospital terciario. Actas Dermosifiliogr. 1996;87:681-6.

13. Guerra-Tapia A, González Barber A. Evaluación del paciente dermatológico en la asistencia urgente. Lesiones cutáneas y prurito. Su tratamiento. En: Medina Asensio J, editor. Manual de urgencias médicas. 2. ㄹ Ed. Madrid: Díaz de Santos; 1996. p. 93-103. 